\title{
Searches for additional Higgs bosons in ATLAS
}

\section{M.Florencia Daneri ${ }^{a, *}$ on behalf of the ATLAS Collaboration}

${ }^{a}$ Universidad de Buenos Aires, Argentina

E-mail: daneriflorencia@gmail.com

The discovery of the Higgs boson with the mass of about $125 \mathrm{GeV}$ completed the particle content predicted by the Standard Model. Even though this model is well established and consistent with many measurements, it is not capable to solely explain some observations. Many extensions addressing such shortcomings introduce additional Higgs-like bosons which can be either neutral, singly-charged or even doubly-charged, or additional resonances with masses larger or smaller than that of the Standard Model Higgs boson. The current status of searches for these particles based on the full LHC Run 2 dataset of the ATLAS experiment at $13 \mathrm{TeV}$ are presented.

*** The European Physical Society Conference on High Energy Physics (EPS-HEP2021), ***

*** 26-30 July $2021 * * *$

*** Online conference, jointly organized by Universität Hamburg and the research center DESY ***

${ }^{*}$ Speaker 


\section{Introduction}

The Higgs boson discovery at the LHC by the ATLAS and CMS Collaborations in 2012 $[2,3]$ and subsequent campaigns of precision measurements of Higgs boson production and decay properties resulted in the establishment of the Standard Model (SM) of particle physics as an effective description of nature up to the TeV energy scale. Nevertheless, the SM is not the ultimate theory of nature since there are serious shortcomings (hierarchy problem, baryon asymmetry or dark matter/energy nature). Many Beyond Standard Model (BSM) theories predict modified and extended Higgs sectors with additional Higgs bosons. Some like the Two-Higgs-Doublet models (2HDM) [4] and Three-Higgs-Doublet models (3HDM) [5] predict neutral and charged Higgs bosons, motivating thus searches for these charged particles. In other scenarios these bosons can decay into a SM Higgs boson pair $(H H)$, leading to resonant production searches. Any deviation from the SM predictions would open a window to new BSM physics, making this searches particularly interesting.

\section{ATLAS search program}

Lots of searches for additional Higgs bosons have been performed by ATLAS [1] with Run 2 dataset in a wide mass range. They target different production and decay modes, and various models are used to interpret the results. Figures $1 \mathrm{a}$ and $1 \mathrm{~b}$ summarize the latest results as a function of the boson mass.

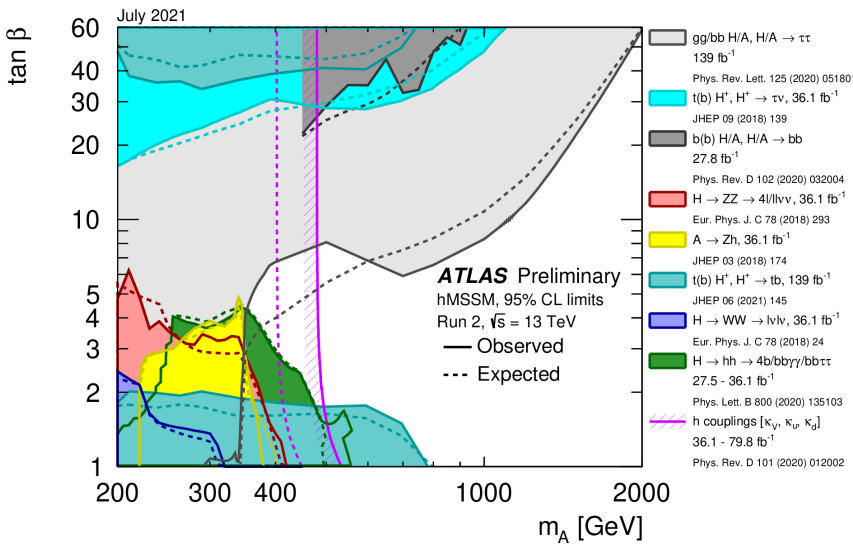

(a)

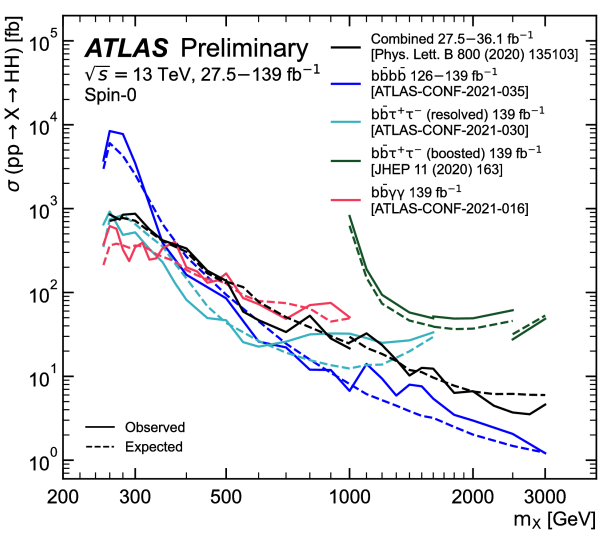

(b)

Figure 1: (a) Regions of the $\left[m_{\mathrm{A}}, \tan \beta\right]$ plane excluded in the hMSSM via direct searches for heavy Higgs bosons and fits to the measured rates of observed Higgs boson production and decays. Limits are quoted at $95 \% \mathrm{CL}$ and are indicated for the data (solid lines) and the expectation for the SM Higgs sector (dashed lines).The light shaded or hatched regions indicate the observed exclusions. (b) Upper limits at $95 \% \mathrm{CL}$ on the resonant $H \mathrm{H}$ production cross-section as a function of the mass for a narrow-width scalar resonance. Results are shown from the statistical combination of the $b \bar{b} \tau^{+} \tau^{-}, b \bar{b} b \bar{b}, b \bar{b} \gamma \gamma, W^{+} W^{-} W^{+} W^{-}, W^{+} W^{-} \gamma \gamma$ and $b \bar{b} W^{+} W^{-}$ searches with $36 \mathrm{fb}^{-1}$ and from the searches using $139 \mathrm{fb}^{-1}$ in the $b \bar{b} \gamma \gamma$, resolved and boosted $b \bar{b} \tau^{+} \tau^{-}$, and $b \bar{b} b \bar{b}$ channels. Images from $[6,7]$.

\subsection{Charged Higgs bosons searches}

\subsection{1 $H^{ \pm} \rightarrow c b$}

In some BSM scenarios, $H^{ \pm}$can be lighter than the top quark $\left(m_{\mathrm{H}^{ \pm}}<m_{\mathrm{t}}\right)$ and can decay predominantly to a charm and a bottom quark, $H^{ \pm} \rightarrow c b$. This is the case for some $2 \mathrm{HDM}$ and 
3HDM models, that feature two charged Higgs bosons and predict a high branching ratio for this particular decay.

For the first time in ATLAS, a dedicated search was carried for this topology using the full Run 2 dataset with $\mathcal{L}=139 \mathrm{fb}^{-1}$ and a center of mass energy of $\sqrt{s}=13 \mathrm{TeV}$ [8]. The search is focused on low mass charged Higgs $\left(60 \leq m_{\mathrm{H}^{ \pm}} \leq 160 \mathrm{GeV}\right)$ from decays in $t \bar{t}$ system with $t \bar{t} \rightarrow W b H^{ \pm} b$.

The signal is characterised by the presence of one isolated electron or muon and at least four jets, with at least two of them $b$-tagged. The main background comes from SM $t \bar{t} \rightarrow W b W b$ processes, and it is split in flavour components $(t \bar{t}+\geq 1 b, t \bar{t}+\geq 1 c$ and $t \bar{t}+l i g h t)$ for a better treatment. Events are categorized in nine regions depending on the number of jets $(4 \mathrm{j}, 5 \mathrm{j}$ and $6 \mathrm{j})$ and the number of $b$-tagged jets $\left(2 \mathrm{~b}+1 \mathrm{bl}{ }^{1}, 3 \mathrm{~b}, 4 \mathrm{~b}\right)$. Considering regions with different jet and $b$-jet multiplicity is particularly useful to isolate regions with different signal to background ratios while at the same time calibrating the $t \bar{t}+$ jet background prediction and constraining the related systematic uncertainties.

Regions with $(2 \mathrm{~b}+1 \mathrm{bl})$ are used to derive data-based corrections to improve the $t \bar{t}$ modeling, while $(4 \mathrm{j}, 3 \mathrm{~b})$ and $(5 \mathrm{j}, 3 \mathrm{~b})$ are considered the main signal regions. Regions with $(6 \mathrm{j}, 3 \mathrm{~b})$ are used to improve background modeling and $(4 \mathrm{j}, 4 \mathrm{~b})$ regions to recover acceptance for signal events with a mistagged $c$-quark. Finally, $(5 \mathrm{j}, \geq 4 \mathrm{~b})$ and $(6 \mathrm{j}, \geq 4 \mathrm{~b})$ regions are control regions dominated by $t \bar{t}+\geq 1 b$ processes.

To discriminate signal and background a feed-forward Neural Network (NN) classifier is built and it is trained with signal events against $t \bar{t}$ events in $\geq 4 \mathrm{j}, 3 \mathrm{~b}$ regions. Jets and lepton kinematics, $b$-tagging information and invariant mass variables are used as input variables for the NN, which is also parametrized as a function of $m_{\mathrm{H}^{ \pm}}$and trained for all signals together.

Finally a binned profile likelihood fit is performed over the discriminant output across $3 \mathrm{~b}$ and $4 \mathrm{~b}$ regions to calibrate the background and reduce the impact of systematic uncertainties. Figure $2 \mathrm{~b}$ shows the NN discriminant after the fitting was performed for $(4 \mathrm{j}, 3 \mathrm{~b})$ regions.

Upper limits on $\mathcal{B}\left(t \rightarrow H^{ \pm} b\right) \times \mathcal{B}\left(H^{ \pm} \rightarrow c b\right)$ at $95 \% \mathrm{CL}$ are derived. As it can be seen in Figure $2 \mathrm{a}$, the observed (expected) limits vary between $0.15 \%(0.09 \%)$ and $0.42 \%(0.25 \%)$ depending on $m_{\mathrm{H}^{ \pm}}$. The largest excess in data is seen at $m_{\mathrm{H}^{ \pm}}=130 \mathrm{GeV}$ with a local (global) significance of $3.0(2.0) \sigma$.

\subsection{Di-Higgs production searches}

\subsection{1 $H H \rightarrow b \bar{b} \tau^{+} \tau^{-}$}

A search for Higgs boson pair produced in gluon-gluon fusion events with two $b$-jets and two $\tau$-leptons is presented using the full Run 2 dataset with $\mathcal{L}=139 \mathrm{fb}^{-1}$ and a center of mass energy of $\sqrt{s}=13 \mathrm{TeV}$ [9]. SM Higgs boson pairs produced in the decay of a narrow-width scalar resonance predicted by $2 \mathrm{HDM}$ in the mass range 251 to $1.6 \mathrm{TeV}$ are targeted. The final state has relatively large branching fraction $(7.3 \%)$ and presents a clean signature.

The signal signature are two $b$-tagged jets and two $\tau$-leptons with opposite charge, and both decay combinations are considered ( $\tau_{\text {had }} \tau_{\text {had }}$ and $\left.\tau_{\text {lep }} \tau_{\text {had }}\right)$. As for the analysis strategy, three categories based on the different decay modes and trigger selections are defined: HadHad, Single

\footnotetext{
${ }^{1}$ Regions with $2 b$-tagged jets passing the nominal $b$-tagging operating point of the analysis of $60 \%$, and $1 b$-tagged jet with a looser operating point of $70 \%$.
} 


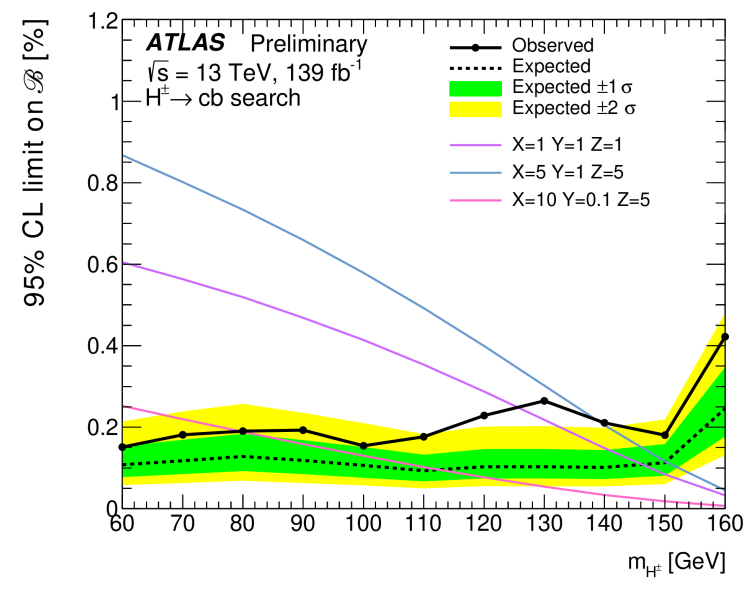

(a)

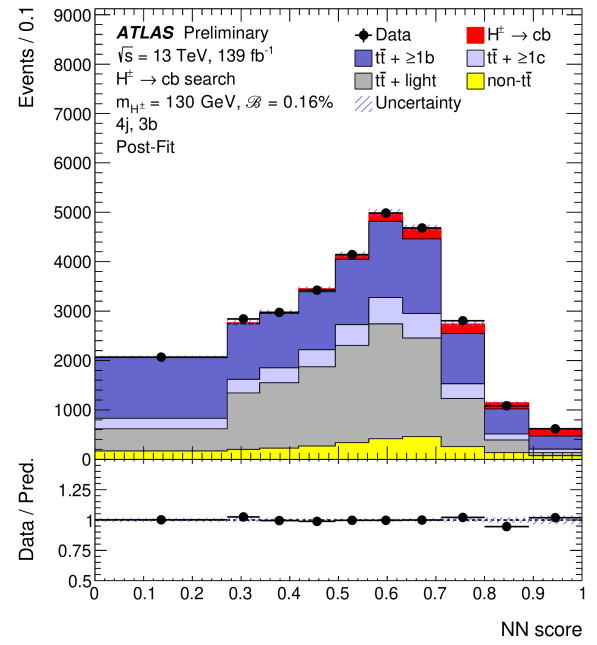

(b)

Figure 2: (a) The observed (solid) $95 \% \mathrm{CL}$ upper limits on $\mathcal{B}\left(t \rightarrow H^{ \pm} b\right) \times \mathcal{B}\left(H^{ \pm} \rightarrow c b\right)$ as a function of $m_{\mathrm{H}^{ \pm}}$and the expectation (dashed) under the background-only hypothesis. (b) Comparison between the data and prediction for the $\mathrm{NN}$ score in the fit region $(4 \mathrm{j}$, 3b) after the likelihood fit to data. The post-fit signal for $m_{\mathrm{H}^{ \pm}}=130 \mathrm{GeV}$ is displayed as a red histogram normalised to the best-fit branching fraction of $0.16 \%$. Plots are taken from [8].

Lepton Trigger (LepHad SLT), Lepton Tau Trigger (LepHad LTT), which translates into three signal regions. HadHad and LepHad SLT categories have about the same acceptance; depending on the mass range, HadHad has even larger acceptance. LepHad SLT has larger acceptance than HadHad below $400 \mathrm{GeV}$.

For the backgrounds, $t \bar{t}$ with $\tau_{\text {had }}$ and $Z \rightarrow \tau \tau+$ HF events are modeled with simulations and their normalizations are determined from control regions in the final fit. For the HadHad category, fake taus coming from $t \bar{t}$ and QCD background processes are estimated from data-driven techniques, using the fake factor method for $t \bar{t}$ and scale factor method for QCD.

The analysis is based in multivariate discriminants (MVA) that are used to extract the signals. A parametric Neural Network is trained separately for the three signal regions and for all signal hypotesis. A binned profile likelihood fit is performed to the MVA scores in the three signal regions and the leptons invariant mass for $\mathrm{Z}+\mathrm{HF}$ events in the control region.

The data are found to be compatible with the background-only hypothesis, with the largest deviation observed at a mass of $1 \mathrm{TeV}$, which corresponds to a local (global) significance of 3.0 $\left(2.0_{-0.2}^{-0.4}\right) \sigma$. As a result, upper limits for the cross section on the resonant di-Higgs production are estimated for the fully hadronic and the hadronic-leptonic channels and also for the combination of both as a function of the scalar mass resonance. Observed (expected) upper limits are set between 26 and $950 \mathrm{fb}$ (12 and $850 \mathrm{fb}$ ) depending on the mass region.

Figure $3 \mathrm{a}$ shows the upper limits as a function of the signal scalar mass and $3 \mathrm{~b}$ the MVA discriminant output for the signal region in the LepHad SLT category.

\subsection{2 $H H \rightarrow b \bar{b} b \bar{b}$}

A di-Higgs production search targeting a $4 b$-tagged jet final state using the full Run 2 dataset with $\mathcal{L}=139 \mathrm{fb}^{-1}$ and a center of mass energy of $\sqrt{s}=13 \mathrm{TeV}$ is presented [10]. Among the 


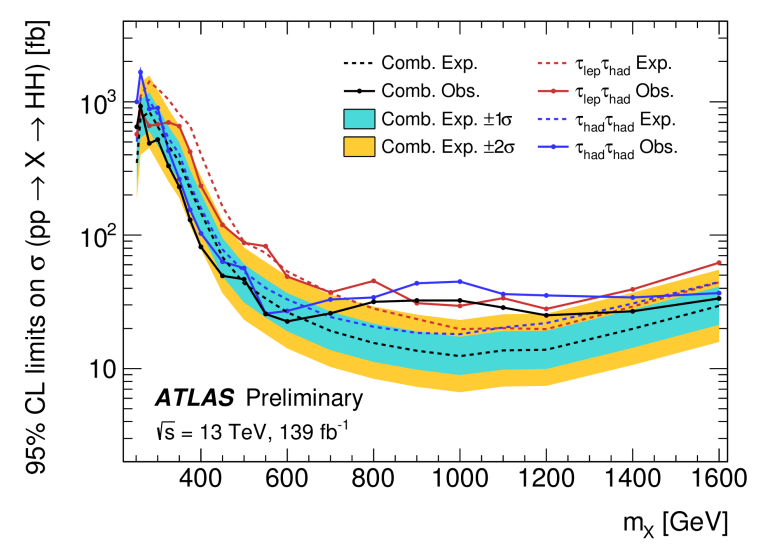

(a)

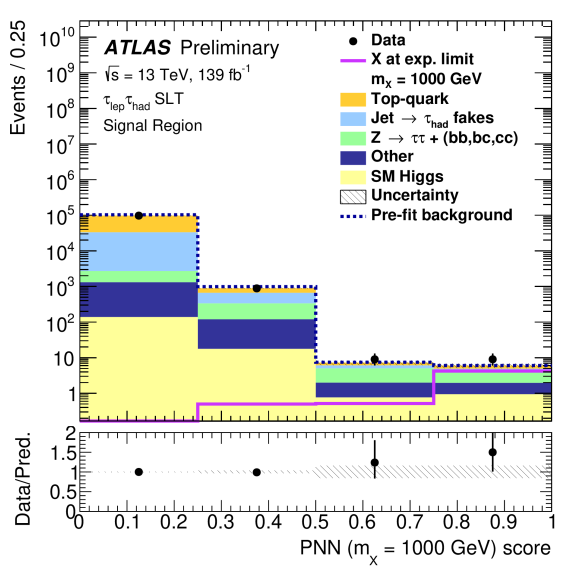

(b)

Figure 3: (a) Observed (solid lines) and expected (dashed lines) limits at 95\% CL on the cross-section of the resonant $H H$ production as a function of the scalar resonance mass for $\tau_{\text {had }} \tau_{\text {had }}$ and $\tau_{\text {lep }} \tau_{\text {had }}$ separately and their combination. (b) The MVA output distribution for resonant $H H$ signal with $m_{\mathrm{X}}=1000 \mathrm{GeV}$ in the LepHad SLT category. The distribution is shown after the fit to the background-only hypothesis is performed. The signal is overlaid and scaled to the combined expected limit. The dashed histogram shows the total pre-fit background. Images from [9].

different decay modes, $b \bar{b} b \bar{b}$ presents the largest branching ratio of about $34 \%$.

Two signal benchmark models are considered for this analysis: a spin-0 narrow width scalar $(X)$ predicted for example in $2 \mathrm{HDM}$ scenarios [4], and a spin-2 Kaluza-Klein graviton $\left(G_{\mathrm{kk}}^{*}\right)$ with $k / \bar{M}_{\mathrm{Pl}}=1$ [11]. Both assumed to be produced via gluon-gluon fusion and decay to two SM Higgs bosons.

The search is split in two complementary channels: resolved, in which each of the four $b$-quarks leads to an individually reconstructed jet; and boosted, which targets the topology where each Higgs boson is produced with large $p_{\mathrm{T}}$ and its decay products are reconstructed as a single large-radius jet. The resolved targets masses between $251 \mathrm{GeV}$ and $1.5 \mathrm{TeV}$, while the boosted focuses on masses from $900 \mathrm{GeV}$ up to $3 \mathrm{TeV}$. The channels are statistically combined in the overlapping region. The main background for this search comes from the QCD multi jets processes.

For both channels, three analysis regions are defined in the two Higgs candidates mass plane. On one side, a control region that is used to estimate the background, then a validation region to asses uncertainties and finally the main signal region that is chosen to optimize the signal significance where the search is performed.

Within the resolved analysis, $2 b$-tagged jets with a radius size of 0.4 are required for each Higgs candidate. The final discriminant variable in this case is the di-Higgs mass $m(H H)$, previously corrected so that the Higgs candidate masses agree with the SM Higgs mass. The background is data driven estimated using a neural network, and it is derived using events with $2 b$-tagged jets.

For the boosted channel, at least two large jets of radius size 1 are required. Events are then categorized according to the multiplicity and $b$-tagging status of the track jets associated with each of the two Higgs candidates. By doing this, three signal-enriched categories with four or fewer $b$-tagged track jets are defined, which are collectively called high-tag categories. While low-tag categories that fail the $b$-tagging requirement are also defined in order to estimate the background. The final discriminant is also $m(H H)$. 
Figures $4 \mathrm{a}$ and $4 \mathrm{~b}$ show $m(H H)$ after the fit is performed in the signal regions for the resolved and boosted channels respectively.

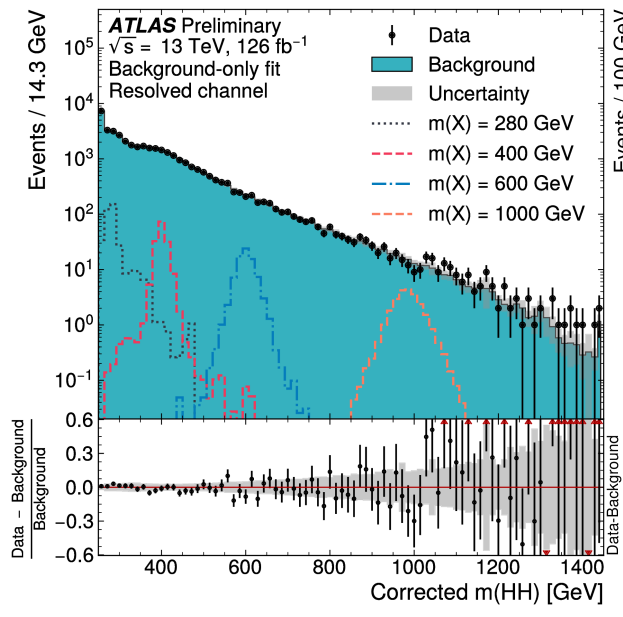

(a)

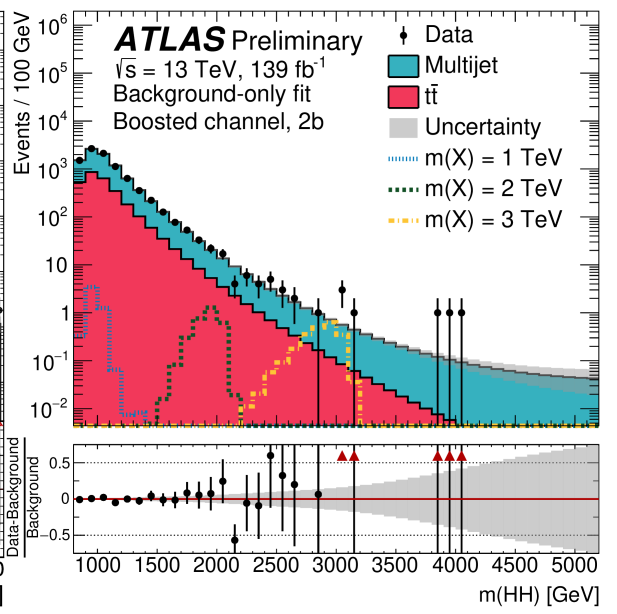

(b)

Figure 4: (a) Corrected $m(H \boldsymbol{H})$ distribution in the resolved $4 \mathrm{~b}$ signal region (dots) and (b) $m(\boldsymbol{H} \boldsymbol{H})$ distribution in the boosted $2 \mathrm{~b}$ signal region (dots), after the fit under the background-only hypothesis. Representative spin-0 signal hypotheses (dashed, dotted, and dashed-dotted lines) are overlaid, normalized to the overall expected limits on their cross-sections. Plots taken from [10].

Since no significant evidence for a signal is observed, upper limits on the production crosssection times branching ratio to Higgs boson pairs for spin-0 and spin- 2 benchmark models are estimated as seen in Figure 5.

The largest excess is observed at a mass of $1100 \mathrm{GeV}$ with a local (global) significance of 2.6 (1.0) $\sigma$ for the spin-0 signal and 2.7 (1.2) $\sigma$ for the spin-2 model. Spin-2 bulk RS model is excluded for graviton masses between $298 \mathrm{GeV}$ and $1440 \mathrm{GeV}$.

The relative reduction in expected cross-section limits with respect to the previous ATLAS search [12] in this final state ranges from approximately $20 \%$ at low resonance masses to more than $80 \%$ at high masses.

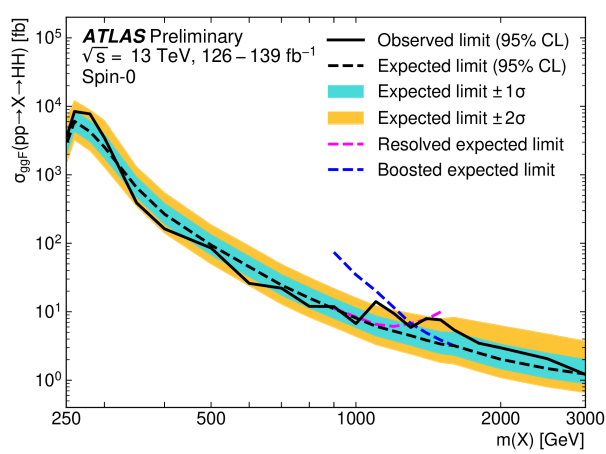

(a)

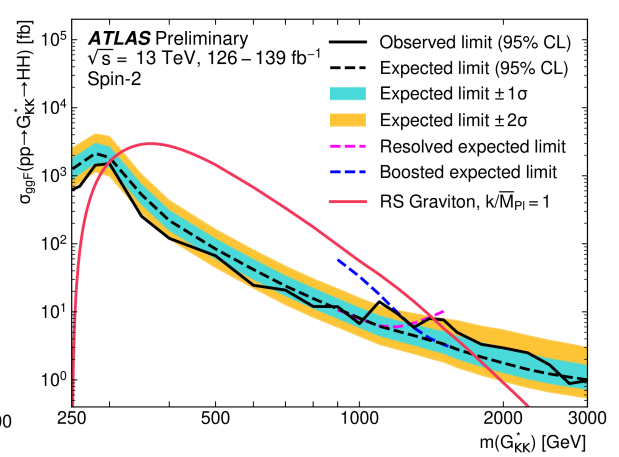

(b)

Figure 5: Expected (dashed black lines) and observed (solid black lines) 95\% CL upper limits on the cross-section times branching ratio of resonant $H H$ production in the (a) spin-0 and (b) spin-2 signal models. Plots taken from [10]. 


\section{Summary}

Some of the latest results for additional Higgs bosons searches in ATLAS based on the full LHC Run 2 dataset are presented, including a search for a light charged Higgs boson $H^{ \pm} \rightarrow c b$ for the first time in ATLAS, as well as searches for di-Higgs production with $b \bar{b} \tau^{+} \tau^{-}$and $b \bar{b} b \bar{b}$ decays that complements other Run-2 results. Better object identification and reconstruction, optimization of analyses: including better background modeling and sequences of (deep) neural networks to better discriminate signals from backgrounds led to improvement beyond the gain from luminosity. No significant excess in data is seen but meaningful updates of constraints on various BSM models are achieved.

\section{References}

[1] ATLAS Collaboration, The ATLAS Experiment at the CERN Large Hadron Collider, JINST 3 (2008) S08003.

[2] ATLAS Collaboration, Observation of a new particle in the search for the Standard Model Higgs boson with the ATLAS detector at the LHC, Phys. Lett. B 716 (2012) 1, [arXiv: 1207.7214].

[3] CMS Collaboration, Observation of a new boson at a mass of $125 \mathrm{GeV}$ with the CMS experiment at the LHC, Phys. Lett. B 716 (2012) 30, [arXiv: 1207. 7235].

[4] G. C. Branco et al., Theory and phenomenology of two-Higgs-doublet models, Phys. Rept.516 (2012) 1, [arXiv: 1106.0034].

[5] A. G. Akeroyd, S. Moretti, K. Yagyu and E. Yildirim, Light charged Higgs boson scenario in 3-Higgs doublet models, Int. J. Mod. Phys. A 32 (2017) 1750145, [arXiv: 1605. 05881].

[6] ATLAS Collaboration, hMSSM summary plots from direct and indirect searches, ATL-PHYSPUB-2021-030 (2021), http: //cdsweb . cern . ch/record/2777012

[7] ATLAS Collaboration, Summary of non-resonant and resonant Higgs boson pair searches from the ATLAS experiment, ATL-PHYS-PUB-2021-031 (2021), http://cdsweb.cern. $\mathrm{ch} / \mathrm{record} / 2777013$

[8] ATLAS Collaboration, Search for a light charged Higgs boson in $t \rightarrow H^{ \pm}$b decays, with $H^{ \pm} \rightarrow$ $c b$, in the lepton+jets final state in proton-proton collisions at $\sqrt{s}=13 \mathrm{TeV}$ with the ATLAS detector, ATLAS-CONF-2021-037 (2021), http: //cdsweb. cern.ch/record/2779169

[9] ATLAS Collaboration, Search for resonant and non-resonant Higgs boson pair production in the $b \bar{b} \tau^{+} \tau^{-}$decay channel using $13 \mathrm{TeV}$ pp collision data from the ATLAS detector, ATLAS-CONF-2021-030 (2021), http: //cdsweb . cern . ch/record/2777236

[10] ATLAS Collaboration, Search for resonant pair production of Higgs bosons in the $b \bar{b} b \bar{b}$ final state using pp collisions at $\sqrt{s}=13 \mathrm{TeV}$ with the ATLAS detector, ATLAS-CONF-2021-035 (2021), http://cdsweb. cern. ch/record/2777861 
[11] A. Oliveira, Gravity particles from Warped Extra Dimensions, predictions for LHC, (2014), [arXiv: 1404.0102].

[12] ATLAS Collaboration, Search for pair production of Higgs bosons in the $b \bar{b} b \bar{b}$ final state using $\sqrt{s}=13 \mathrm{TeV}$ with the ATLAS detector, JHEP 01 (2019) 030 [arXiv: 1804.06174] 